\title{
LOW-SAMPLING-RATE M-ARY MULTIPLE ACCESS UWB COMMUNICATIONS IN MULTIPATH CHANNELS
}

\author{
Mohammad T. Alkhodary ${ }^{1}$, Tarig Ballal ${ }^{2}$, Tareq Y. Al-Naffouri ${ }^{1,2}$, and Ali H. Muqaibel ${ }^{1}$ \\ ${ }^{1}$ Electrical Engineering Department, King Fahd University of Petroleum \& Minerals \\ ${ }^{2}$ Electrical Engineering Department, King Abdullah University of Science \& Technology
}

\begin{abstract}
The desirable characteristics of ultra-wideband (UWB) technology are challenged by formidable sampling frequency, performance degradation in the presence of multi-user interference, and complexity of the receiver due to the channel estimation process. In this paper, a low-rate-sampling technique is used to implement $M$-ary multiple access UWB communications, in both the detection and channel estimation stages. A novel approach is used for multiple-access-interference (MAI) cancelation for the purpose of channel estimation. Results show reasonable performance of the proposed receiver for different number of users operating many times below Nyquist rate.
\end{abstract}

Index Terms - Low Sampling, Channel Estimation, Ultra-Wideband, Multiple-access-interference cancelation

\section{INTRODUCTION}

Ultra-wideband (UWB) technology is promising for wireless communication with desirable characteristics such as low-power consumption, high bandwidth, and shared spectral resources with co-existing systems. UWB applications vary from short-range communications with high data-rate to long-range communications with low data-rate [1].

Impulse radio UWB communications uses ultra-short duration pulses, typically on the order of nanoseconds, to convey information. Several benefits are attained by transmitting such ultra-short pulses. First, the simplicity of the transmitter design, since a carrier-less baseband signal is used for carrying information. Second, the transmitted power is distributed over a wide-bandwidth, which results in a weak or no impact on the other narrow-band wireless systems sharing the same spectrum [1].

The design of UWB receiver is met with several challenges such as interference cancellation, high demand on analog to digital converters, channel estimation, timing synchronization, among others. Coherent-based receivers were proposed to exploit the energy spread over large number of channel taps. This demands a large number of rake receiver fingers, and formidable sampling frequency in excess of 25 $\mathrm{GHz}$, especially when it comes to the channel estimation stage [2]. Non-coherent receivers are an alternative suggestion for avoiding such demand. For instance, an energy detection and autocorrelation receiver that performs signal detection in the analog domain was proposed in [3]. Although the design of analog receivers is quite simple, they are limited in performance when compared to their digital counterparts.

Several studies cast the problem of high sampling rate and channel estimation into compressive sensing (CS) framework $[4,5]$. Symbol detection techniques based on CS were proposed in [4], where the received signal is sampled below the Nyquist rate. The theory of CS states that the number of samples that are required to recover the signal is proportional to the sparsity of the signal in some "dictionary" domain. Hence, sparsity is essential for all CS techniques. Despite the achieved reduction in sampling frequency, the sparsity assumption is not always realistic in practical situations.

A low-sampling technique was proposed in [6] and [7] for UWB digital receivers using equivalent time sampling. The received UWB signals were sampled many-times below Nyquist rate. The process starts at the transmitter, where the desired symbol is transmitted repetitively for $R$ times. The receiver samples the received signal at $f_{N} / R$, where $f_{N}$ is the required Nyquist sampling frequency. The technique was applied to perform channel estimation where the receiver sampled the received signal 20 times below Nyquist rate, regardless of the sparsity level of the signal. It was shown that the receiver is able to detect binary symbols for a single user with the same sub-sampling rate.

Motivated by the work in $[6,7]$, this paper applies the low-sampling technique to $M$-ary multiple access UWB communications. We use the widely implemented pulse position modulation (PPM) scheme along with time hopping (TH) as a multiple access technique. Orthogonal codes are assigned to each user to aid the receiver in multiple-access-interference (MAI) cancellation during the channel estimation stage.

The remaining of this paper is organized as follows. Section 2 presents the signal model. In section 3 , we elaborate on the detection process using sub-Nyquist sampling. Channel estimation is discussed in section 4. Finally, we present the bit-error-rate (BER) of the proposed system in section 5 . 


\section{SIGNAL MODEL}

\subsection{Transmit Signal Model (Continuous-Time)}

The proposed UWB system uses TH multiple access to differentiate between users [8]. PPM is used for data symbol modulation [9]. The user sends a data symbol $i$ where $i \in$ $\{0,1, \ldots, M-1\}, M=2^{b}, b$ is the number of bits in one symbol. A transmitted data symbol of one user, is composed of $N_{s}$ frames ${ }^{1}$ each of duration $T_{f}$. A frame is divided into $N_{c}$ chips each of duration $T_{c}$. To transmit one data symbol, the user sends $N_{s}$ pulses consecutively, i.e., one pulse per frame. The location of the pulse inside the frame is user and symbol dependent. Fig.1 illustrates the transmitted waveform of one symbol.

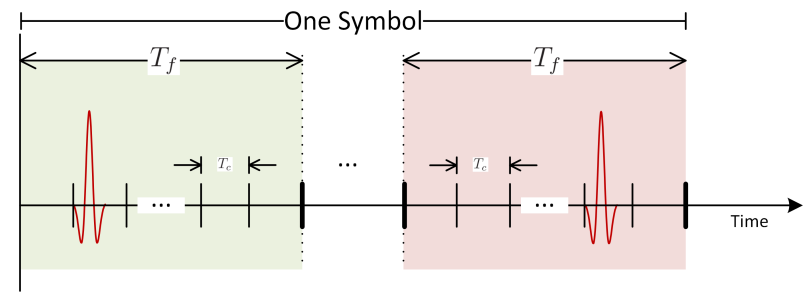

Fig. 1: Illustration of one transmitted symbol.

Accordingly, the transmitted signal for the $v^{\text {th }}$ user can be expressed as

$$
x_{i}^{(v)}(t)=\sum_{k=0}^{N_{s}-1} g\left(t-k T_{f}-c_{k}^{(v)} T_{c}-\delta_{i(v)}^{k}\right),
$$

where $g(t)$ is the second derivative of the well-known Gaussian pulse [9] of a duration $T_{g}$ where $T_{g} \ll T_{c} . k$ is the index of the transmitted pulses and indicates the number of time hops in one data symbol. Assuming that the number of users is $N_{u}, v$ has a range of $1 \leq v \leq N_{u}$. The sequence $\left\{c_{k}^{(v)}\right\}$ is a pseudorandom time-hopping sequence that is assigned to the $v^{\text {th }}$ user. $\left\{c_{k}^{(v)}\right\}$ is periodic with a period of $N_{p}$, i.e. $c_{k}^{(v)}=c_{k+l N_{p}}^{(v)}$, where $l$ is an integer number. Each element of the hopping sequence is an integer random variable drawn from a uniform distribution over the interval $\left(0, N_{h}\right)$. Hence, the time hopping sequence adds a discrete time shift to the signal with value of $0 \leq c_{k}^{(v)} T_{c} \leq N_{h} T_{c}$.

The time shift $\delta_{d^{(v)}}^{k}$ is used for PPM. Each data symbol is uniquely identified by the sequence of the time shifts $\left\{\delta_{i}^{k}\right\}, k=0,1,2, \ldots, N_{s}-1$, and $i$ is the symbol index. Different methods for choosing the modulation time shifting sequence were proposed and discussed in [9]. In this work, the modulation time shifting sequence has been chosen such that all $M$ signals are mutually orthogonal. This can be done by choosing $\delta_{i}^{k}=2[(k+i-1) \bmod M] T_{g}$.

To avoid the overlapping of pulses from two different frames, the frame time is set to be $T_{f} \geq T_{g}+\delta_{M-1}^{(v)}+N_{h} T_{c}$, where

\footnotetext{
${ }^{1}$ The greater the value of $N_{s}$ the better the detection, and the lower the data rate.
}

$\delta_{M-1}^{(v)}$ is the maximum modulation shift and $N_{h}$ is the maximum shift caused by the hopping sequence.

\subsection{Received Signal Model (Continuous-Time)}

The received signal of the $v^{\text {th }}$ user can be expressed as

$$
r_{i}^{(v)}(t)=x_{i}^{(v)}(t) * h^{(v)}(t)+\ddot{x}(t)+n(t),
$$

where $n(t)$ is an additive white Gaussian noise (AWGN) with zero mean and variance $\sigma^{2}$, while $\ddot{r}(t)$ is the UWB interference caused by other users. This interference is given by

$$
\ddot{x}(t)=\sum_{\substack{j=1 \\ l \neq v}}^{N_{u}} x_{j}^{(l)}\left(t-\tau^{(l)}\right) * h^{(l)}(t),
$$

where $\tau^{(l)}$ is the time synchronization error, and $h^{(v)}$ is the channel of the $v^{\text {th }}$ user.

\subsection{Discrete-Time Model of the Received Signals}

The discrete version of the continuous time signal model in equation (2) is given by

$$
\mathbf{r}_{i}^{(v)}=\mathbf{X}_{i}^{(v)} \mathbf{h}^{(v)}+\ddot{\mathbf{x}}+\mathbf{n} \quad i \in\{0,1, \ldots, M-1\},
$$

where $\mathbf{h}^{(v)} \in \mathbb{R}^{N \times 1}$ is the channel impulse response of the $v^{\text {th }}$ user, $\mathbf{X}_{i}^{(v)} \in \mathbb{R}^{N \times N}$ is the transmission matrix that contains shifted versions of the $i^{\text {th }}$ symbol of the $v^{\text {th }}$ user and it is a Toeplitz matrix, $\mathbf{n}$ is AWGN, and $\ddot{\mathbf{x}}$ is the interference caused by other users, which is given by

$$
\ddot{\mathbf{x}}=\sum_{\substack{l=1 \\ l \neq v}}^{N_{u}} \mathbf{X}_{j}^{(l)} \mathbf{h}^{(l)} \quad j \in\{0,1, \ldots, M-1\} .
$$

\section{DETECTION AT SUB-NYQUIST SAMPLING RATES}

\subsection{Conventional Correlation Detection}

Due to the time hopping and PPM, introduced in section 2.1, each data symbol of a user will have a unique transmission matrix, $\mathbf{X}_{i}^{(v)}$. These matrices, along with the estimated channel impulse response $\hat{\mathbf{h}}^{(v)}$, are used in a correlation receiver for the detection purpose [6]. To decide on the received symbol, the receiver measures the correlation between the received signal in (4) and the symbols' transmission matrices multiplied by the estimated channel. The correlation is obtained as follows

$$
\begin{aligned}
\rho_{j, i}^{(v)} & =\hat{\mathbf{h}}^{(v)^{T}} \mathbf{X}_{j}^{(v)^{T}} \mathbf{r}_{i}^{(v)}, \quad j=0,1, \ldots, M-1 \\
& =\hat{\mathbf{h}}^{(v)^{T}}\left[\mathbf{X}_{j}^{(v)^{T}} \mathbf{X}_{i}^{(v)} \mathbf{h}^{(v)}+\mathbf{X}_{j}^{(v)^{T}} \ddot{\mathbf{x}}+\mathbf{X}_{j}^{(v)^{T}} \mathbf{n}\right],
\end{aligned}
$$

where $(.)^{T}$ indicates the matrix transpose operation. The receiver then decides on the received symbol as the one that produces the maximum correlation $\rho_{j . i}^{(v)}$. 


\subsection{Sub-Sampled Correlation Detection}

A sub-Nyquist sampling technique was proposed in [6] for UWB communications. The transmitter repeats the transmitted pulse $R$ times, the sampling frequency at the receiver will be reduced to $f_{N} / R$, where $f_{N}$ is the Nyquist frequency. The key condition for this process to work successfully is that the number of samples of the repeated signal and $R$ are co-prime numbers.

In this work, we apply the sampling technique proposed in [6] to the case of $M$-ary multi-user UWB communication. Suppose that the transmitter sends $R$ repetitive versions of (1). The transmitted batch is then given by

$$
\bar{x}_{i}^{(v)}(t)=\sum_{a=0}^{R-1} \sum_{k=0}^{N_{s}-1} g\left(t-k T_{f}-c_{k}^{(v)} T_{c}-\delta_{i(v)}^{k}-a T_{s}\right),
$$

where, $a$ is the repetition index. Consequently, the repetitive version of the linear model of the received signal in (4) becomes

$$
\overline{\mathbf{r}}_{i}^{(v)}=\overline{\mathbf{X}}_{i}^{(v)} \mathbf{h}^{(v)}+\overline{\ddot{\mathbf{x}}}+\overline{\mathbf{n}},
$$

where $\overline{\mathbf{X}}_{i}^{(v)}$ represents $R$ concatenated versions of the transmission matrix $\mathbf{X}_{i}^{(v)}$ and it is expressed as

$$
\overline{\mathbf{X}}_{i}^{(v)}=\left[\mathbf{X}_{i_{0}}^{(v)^{T}}, \mathbf{X}_{i_{1}}^{(v)^{T}}, \ldots, \mathbf{X}_{i_{R-1}}^{(v)}\right]^{T}
$$

The term $\overline{\ddot{x}}$ in (8) represents the interference from other users (each user uses the same repetition concept as in (7)) and is given by

$$
\overline{\ddot{\mathbf{x}}}=\sum_{\substack{l=1 \\ l \neq v}}^{N_{u}} \overline{\mathbf{X}}_{j}^{(l)} \mathbf{h}^{(l)}
$$

The received signal in (8) will be sampled at the new subNyquist frequency, $f_{N} / R$. The sub-sampled signal can be expressed as

$$
\tilde{\mathbf{r}}_{i}^{(v)}=\tilde{\mathbf{X}}_{i}^{(v)} \mathbf{h}^{(v)}+\tilde{\ddot{\mathbf{x}}}+\tilde{\mathbf{n}},
$$

where $\tilde{\mathbf{X}}_{i}^{(v)}$ indicates the sub-sampled version of the corresponding symbol in (8). Note that the channel impulse responses $\mathbf{h}$ in (8), (10), and (11) are sampled at Nyquist rate as well as the rows of the transmission matrices. The noise $\overline{\mathbf{n}}$ is a concatenation of $R$ statistically equivalent vectors. Hence, the sub-sampled version of the noise, $\tilde{\mathbf{n}}$, is equivalent to one repetition of $\overline{\mathbf{n}}$

According to Theorem 1 in [6], if $R$ and the number of samples in $\overline{\mathbf{r}}_{i}$ are co-prime, then the sub-sampled signal, $\tilde{\mathbf{r}}_{i}^{(v)}$ and the the received signal $\mathbf{r}_{i}^{(v)}$, as in (4), are equivalent in terms of having the same elements but with different order and different version of noise. Consequently, the following relationship hold true:

$$
\mathbf{X}_{i}^{(v)^{T}} \mathbf{X}_{j}^{(v)}=\tilde{\mathbf{X}}_{i}^{(v)^{T}} \tilde{\mathbf{X}}_{j}^{(v)}, \quad \forall i, j .
$$

Therefore, the correlation parameter in the sub-sampling receiver will become

$$
\begin{aligned}
\tilde{\rho}_{j, i}^{(v)} & =\hat{\mathbf{h}}^{(v)^{T}} \tilde{\mathbf{X}}_{j}^{(v)^{T}} \tilde{\mathbf{r}}_{i}^{(v)}, \quad i=0,1, \ldots, M \\
& =\hat{\mathbf{h}}^{(v)^{T}}\left[\tilde{\mathbf{X}}_{j}^{(v)^{T}} \tilde{\mathbf{X}}_{i}^{(v)} \mathbf{h}^{(v)}+\tilde{\mathbf{X}}_{j}^{(v)^{T}} \ddot{\mathbf{X}}+\tilde{\mathbf{X}}_{j}^{(v)^{T}} \tilde{\mathbf{n}}\right]
\end{aligned}
$$

The receiver will then decide the received symbol based on the maximum value of $\tilde{\rho}_{j, i}^{(v)}$ for all $j=0,1, \ldots, M$.

\section{CHANNEL ESTIMATION}

In this section we first introduce orthogonal pilot symbols that are used to eliminate MAI for aiding channel estimation. Then we elaborate on the bounded data uncertainty [6] estimator that is used to estimate the UWB channel for each user individually.

\subsection{Pilot Symbols Design}

Recall the received signal in (4). The user interference term $\ddot{\mathbf{x}}$ makes the channel estimation, for the $v^{\text {th }}$ user, a challenging task. Our aim, in this section, is to design pilot symbols that would eliminate the user interference term to aid the channel estimation algorithm.

Let us consider $M$ users (i.e. $N_{u}=M$ ) who are assigned $M$ mutually orthogonal codes drawn from columns of a Hadamard matrix of order $M$. Such matrix has the following structure:

$$
\mathbf{W}_{M}=\left[\begin{array}{lllll}
\mathbf{w}^{(0)} & \ldots & \mathbf{w}^{(v)} & \ldots & \mathbf{w}^{\left(N_{u}-1\right)}
\end{array}\right],
$$

where $\mathbf{w}^{(v)}$ is the $v^{\text {th }}$ column of $\mathbf{W}_{M}$ and its elements are $w_{i}^{(v)} \in\{-1,1\}, i=1,2, \ldots, M$. The columns of the matrix $\mathbf{W}_{M}$, referred to as "Walsh codes" [10], satisfy the following orthogonal property:

$$
\mathbf{w}^{(v)^{T}} \mathbf{w}^{(l)}=\left\{\begin{array}{cc}
M & v=l \\
0 & v \neq l
\end{array} \forall v, l \in\{0,1, \ldots, M-1\} .\right.
$$

In order to take advantage of the orthogonality of Walsh codes, each user transmits a unique pilot symbol, $d^{(v)} \in$ $\{0,1, \ldots, M-1\}, M$ times. The symbol is multiplied with the corresponding Walsh item $w_{i}^{(v)}$. Hence, the transmitted pilot symbol can be expressed as

$$
p^{(v)}(t)=w_{m}^{(v)} \sum_{k=0}^{N_{s}-1} g\left(t-k T_{f}-c_{k}^{(v)} T_{c}-\delta_{d^{(v)}}^{k}\right) .
$$

Note that the subscript $d^{(v)}$ indicates that the $v^{\text {th }}$ user chooses the $v^{\text {th }}$ symbol from the symbol set $d^{(v)} \in\{0,1, \ldots, M-$ $1\}$ as a pilot symbol.

Similar to (4), the linear model of the received pilot symbol can be written as

$\mathbf{p}_{m}^{(v)}=w_{m}^{(v)} \mathbf{X}_{d^{(v)}}^{(v)} \mathbf{h}^{(v)}+\ddot{\mathbf{p}}_{m}+\mathbf{n}, \quad m \in\{0,1, \ldots, M-1\}$, 
where $\mathbf{X}_{v}^{(v)}$ is the transmission matrix of the pilot symbol for the $v^{\text {th }}$ user.

Assuming that all users are synchronized and transmit their pilot symbols simultaneously, then $\ddot{\mathbf{p}}$, the interference from the pilot symbols of the other users, is given by

$$
\ddot{\mathbf{p}}_{m}=\sum_{\substack{l=1 \\ l \neq v}}^{N_{u}} w_{m}^{(l)} \mathbf{X}_{d^{(l)}}^{(l)} \mathbf{h}^{(l)} .
$$

The receiver will multiply the received pilot symbols with their corresponding Walsh codes and add them all together. This yields:

$$
\begin{aligned}
\mathbf{p}^{(v)} & =\sum_{m=0}^{M-1} w_{m}^{(v)} w_{m}^{(v)} \mathbf{X}_{v}^{(v)} \mathbf{h}^{(v)}+\underset{w}{(v)} \ddot{\mathbf{p}_{m}}+w_{m}^{(v)} \mathbf{n} \\
& =M \mathbf{X}_{v}^{(v)} \mathbf{h}^{(v)}+M \mathbf{n} .
\end{aligned}
$$

Due to the orthogonality property of Walsh codes, MAI in (19) will be canceled out.

To apply the sub-sampling technique in the channel estimation stage, the pilot symbol in (16) is transmitted repetitively for $R$ times, then we end up with the matrix modeled in (9). The signals are then sampled at a rate of $f_{N} / R$. The summation of the sub-sampled pilot symbols can be expressed as

$$
\tilde{\mathbf{p}}^{(v)}=M \tilde{\mathbf{X}}_{d^{(v)}}^{(v)} \mathbf{h}^{(v)}+M \tilde{\mathbf{n}} .
$$

The sub-sampled discrete signal $\tilde{\mathbf{p}}^{(v)}$ is passed to the channel estimation algorithm in order to estimate the channel impulse responses of each user.

\subsection{Channel Estimation Algorithm}

A channel estimation technique is derived in $[6,7]$ using a bounded data uncertainty (BDU) approach. The final estimator can be implemented in a computationally efficient manner using the fast Fourier transform (FFT) and its inverse (IFFT). The channel estimator is given by

$$
\hat{\mathbf{h}}^{(v)}=\operatorname{ifft}\left[\left(\Sigma^{(v)^{2}}+\gamma \mathbf{I}\right)^{-1} \mathrm{fft}\left(\tilde{\mathbf{X}}_{d^{(v)}}^{(v)^{T}} \tilde{\mathbf{p}}^{(v)}\right)\right]
$$

where $\Sigma^{(v)}$ is the singular-values matrix of $\tilde{\mathbf{X}}_{d^{(v)}}^{(v)}$. The parameter $\gamma$ can be computed by solving

$$
\tilde{\mathbf{p}}^{(v)^{T}} U\left(\Sigma^{(v)^{2}}-\eta^{2} \mathbf{I}\right)\left(\Sigma^{(v)^{2}}+\gamma I\right)^{-2} U^{T} \tilde{\mathbf{p}}^{(v)}=0 .
$$

The solution of (22) and the ways of choosing the parameter $\eta$ are described in [6,7], where the estimator in (21) was shown to significantly outperform to the least squares estimator.

\section{RESULTS AND DISCUSSIONS}

In this section, we present the simulation results for the proposed low-complexity UWB communication approach. We used the IEEE 802.15.4a standard [11] to generate the channel impulse responses based on UWB LOS indoor channel models (CM1). For this model, the maximum excess delay was estimated to be $8 \mathrm{~ns}$ at $-10 \mathrm{~dB}$ and $80 \mathrm{~ns}$ at $-60 \mathrm{~dB}$ [1]. The transmitted pulse was the second derivative of Gaussian pulse with $1 \mathrm{~ns}$ duration. The Nyquist rate was $4 \mathrm{GHz}$ for $-10 \mathrm{~dB}$ bandwidth. The results presented in this section were obtained by averaging over 500 simulation trials. Each trial consisted of the transmission of 300 random symbols for each user, drawn uniformly from the symbol set $\{0,1, \ldots, M-1\}$, resulting in a total of $150 \mathrm{~K}$ symbols for each user in all trials. The channels were generated independently for each user and assumed to be stationary during the transmission time. Each user transmitted $M$ pilot symbols that were coded by the user's Walsh code for MAI cancellation. The estimated channels were then used for symbol detection as in (13).

The transmitted signals were constructed at the Nyquist rate and then convolved with the channel impulse responses, to obtain the noise-free received signal. Each transmitted signal comprised $R$ repetition of symbols. At the receiver side, additive white noise was added to the received signal and finally the signal was down-sampled by a factor of $R$.

Performance was measured by calculating the bit error rate (BER) for different scenarios. Fig. 2 and Fig. 3 plot the BER versus the signal to noise ratio (SNR) for different values of the sub-sampling factor $R$. Fig. 2 shows the performance when the number of users, and the number of data symbols were four, i.e. $N_{u}=M=4$. The performance is comparable for all sub-sampling cases, except when the number of signal repetitions $R=5$ is not co-prime with the number of samples in the received signal, which equals to, in this case, 1930 samples. In such case, the receiver fails to pick the right samples to represent the original signal sampled at Nyquist rate. Fig. 3 shows the system performance for number of users $N u=8$. The degradation in the sub-sampled case is due to performance loss related to the BDU estimator [7]. The effect of user interference is clearly shown in Fig. 4 where the performance for different number of users is shown. The repetition rate was equal to $R=7$. Consequently the sampling frequency was $f_{s}=571.4 \mathrm{MHz}$.

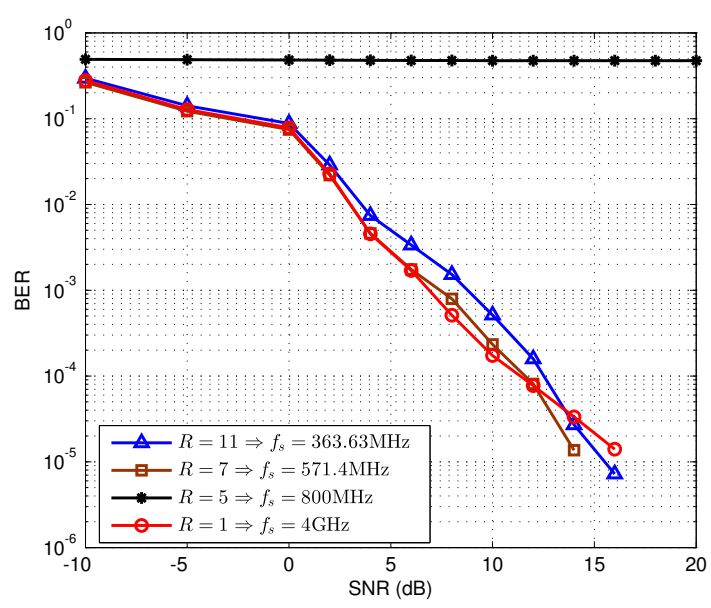

Fig. 2: System performance for number of users, $N_{u}=4$ 


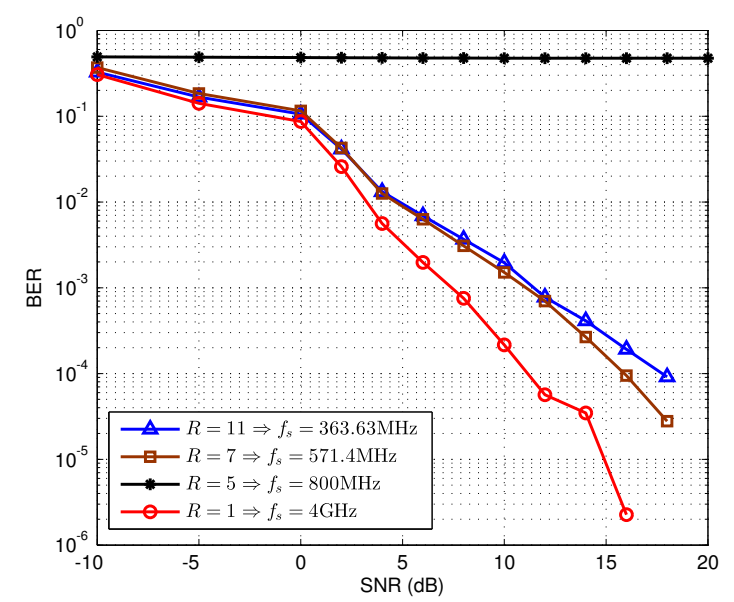

Fig. 3: System performance for number of users, $N_{u}=8$

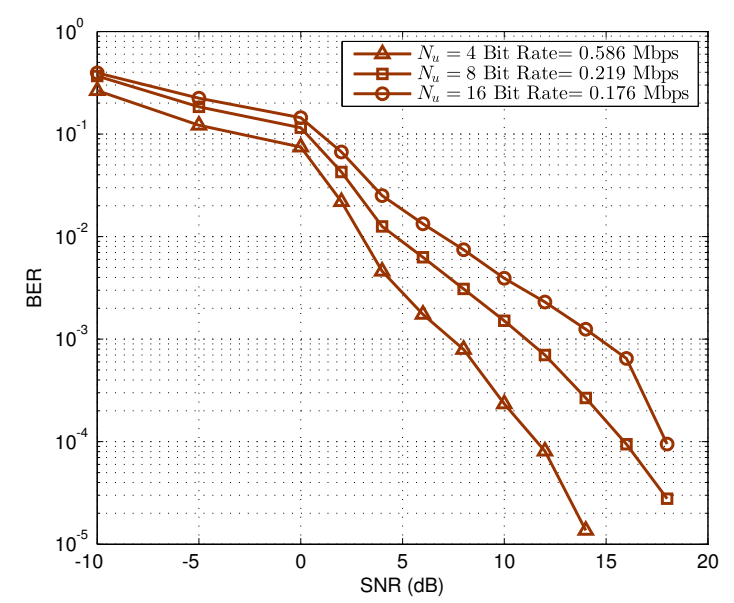

Fig. 4: System performance for different number of users at repetition rate, $R=7$

\section{CONCLUSION}

A low-sampling-rate system for M-ary multiple access UWB communications has been proposed. The system exploits multiple observations generated by transmitting multiple symbols. In the proposed system, we transmit the desired data symbols repetitively to reduce the sampling rate at the receiver. The key condition for this scheme to work successfully is that the number of samples in the discrete version of the signal should be co-prime with the number of symbol repetition. A bounded data uncertainty estimator was applied to estimate the channel impulse response. A novel approach was proposed for multiple-access interference cancelation that aids channel estimation. Multi-user interference was handled by means of orthogonal codes used as pilot symbols for different users. Results were presented, for different number of users. For the case of four users, the performance of the receivers, using sampling frequencies of $1 / 7^{\text {th }}$ and $1 / 11^{\text {th }}$ of the Nyquist frequency, was comparable. Performance slightly degrades for 8 and 16 users.

\section{ACKNOWLEDGMENT}

The authors would like to acknowledge the support provided by King Fahd University of Petroleum and Minerals (KFUPM) and King Abdullah University of Science and Technology (KAUST) for funding this work through the research institute project number EE002355

\section{REFERENCES}

[1] M. Benedetto, T. Kaiser, A. Molisch, I. Oppermann, and C. Politano, UWB Communication Systems A Comprehensive Overview, Hindawi, 2006.

[2] V. Lottici, A D'Andrea, and U. Mengali, "Channel estimation for ultra-wideband communications," Selected Areas in Communications, IEEE Journal on, vol. 20, no. 9, pp. 1638-1645, Dec. 2002.

[3] K. Witrisal, G. Leus, G.J.M. Janssen, M. Pausini, F. Troesch, T. Zasowski, and J. Romme, "Noncoherent ultra-wideband systems," Signal Processing Magazine, IEEE, vol. 26, no. 4, pp. 48-66, Jul. 2009.

[4] A.H. Muqaibel and M.T. Alkhodary, "Practical application of compressive sensing to ultra-wideband channels," Communications, IET, vol. 6, no. 16, pp. 2534 2542, Nov. 2012.

[5] X. Cheng, M. Wang, and Y.L. Guan, "Ultra-wideband channel estimation: A bayesian compressive sensing strategy based on statistical sparsity," Vehicular Technology, IEEE Transactions on, vol. PP, no. 99, pp. 1-1, 2014.

[6] T. Ballal and T.Y. Al-Naffouri, "Low-sampling-rate ultra-wideband channel estimation using equivalenttime sampling," Signal Processing, IEEE Transactions on, vol. 62, no. 18, pp. 4882-4895, Sep. 2014.

[7] T. Ballal and T.Y. Al-Naffouri, "Low-sampling-rate ultra-wideband digital receiver using equivalent-time sampling," in Ultra-WideBand (ICUWB), 2014 IEEE International Conference on, Sep. 2014, pp. 321-326.

[8] F. Kharrat-Kammoun, C.J. Le Martret, and P. Ciblat, "Performance analysis of ir-uwb in a multi-user environment," Wireless Communications, IEEE Transactions on, vol. 8, no. 11, pp. 5552-5563, Nov. 2009.

[9] F. Ramirez-Mireles, "Performance of ultrawideband ssma using time hopping and m-ary ppm," Selected Areas in Communications, IEEE Journal on, vol. 19, no. 6, pp. 1186-1196, Jun. 2001.

[10] K. Eshima, Y. Hase, S. Oomori, F. Takahashi, and R. Kohno, "M-ary uwb system using walsh codes," in Ultra Wideband Systems and Technologies, 2002. Digest of Papers. 2002 IEEE Conference on, May 2002, pp. 37-40.

[11] F. Molisch, Kannan Balakrishnan, Chia chin Chong, Shahriar Emami, Andrew Fort, Johan Karedal, Juergen Kunisch, Hans Schantz, Ulrich Schuster, and Kai Siwiak, "Ieee 802.15.4a channel model - final report," 2004. 